\title{
TEXTURES AND GALAXY FORMATION
}

\author{
DAVID N. SPERGEL \\ Princeton University Observatory \\ Princeton, NJ 08540 USA
}

\begin{abstract}
Textures will produce positively skewed non-Gaussian primordial fluctuations. This implies that galaxies and quasars formed earlier in this model than in a Gaussian model with the same power spectrum. The model also predicts the existence of more high velocity dispersion clusters and richer superclusters. We describe the texture model and our numerical simulations of the origin of structure in the model.
\end{abstract}

\section{What is texture?}

Global texture (Turok 1989) is the latest of a set of theories based on a simple physical idea: the universe began in a hot, homogeneous state and then, as it cooled, underwent a symmetry breaking phase transition that led to the formation of topological defects (Kibble 1976). These defects induced perturbations that seeded the formation of galaxy and large-scale structure. In this scenario, there are short-lived seeds on all scales that persist just long enough to leave an imprint as compensated perturbations in the matter and radiation density.

The texture model has the advantage of being highly predictive. It has only one free parameter, the scale of symmetry breaking, which we normalize to the amplitude of the power spectrum. A specific feature of the texture-seeded cosmologies is the highly non-Gaussian nature of fluctuations produced through the continuous formation of horizon-sized texture 'knots'. The knots collapse down to an infinitesimal scale (the inverse GUT scale) and unwind themselves. In the process they attract the surrounding dark matter and produce a roughly spherical infall pattern. This leads to the early formation of nonlinear objects and highly skewed initial fluctuations.

While the initial power spectrum in a texture-seeded cosmology is very similiar to that of the Harrison-Zel'dovich models, such as inflation, the non-Gaussian nature of the fluctuations leads to distinctive scenario for galaxy formtion and the origin of large scale structure.

\section{Galaxy Formation in the Texture Model}

Galaxy formation in the texture model begins early. In Gooding et al. (1991), we used an analytical model to sudy the evolution of the texture-induced fluctuations. We assumed that each texture knot collapse produced a density fluctuations and used the analytical texture evolution solution (Turok and Spergel 1990), together with the spherical collapse model (Gunn and Gott 1972, Peebles 1980) to compute the evolution of these density fluctuations. We found that by $z \sim 50,6 \times 10^{-5}$ of the mass of the universe has formed non-linear objects of mass greater than $10^{6} \mathrm{M}_{\odot}-$ these objects may have reionized the universe. Most objects larger than $10^{11} \mathrm{M}_{\odot}$ form by $z \sim 2-3$, consistent with the observed epoch of QSO formation. Since these fluctuations are compensated, there is little late accretion of material onto galaxies in the model. This contrasts with the standard CDM model, in which late infall of massive satellites threatens the integrity of galactic disks (Toth and Ostriker 1991).

The analytical estimates of galaxy properties in the texture model show a remarkable agreement with observations. If we assume a mass-to-light ratio of $100 M_{\odot} / L_{\odot}$ for an $L_{*}$ galaxy, the texture model predicts circular velocity, $v_{\text {circ }}=v_{L O S} \sqrt{2}$ of $287 \mathrm{~km} / \mathrm{s}$ for a $b=1$ model, $216 \mathrm{~km} / \mathrm{s}$ for a 
$b=2$ model and $185 \mathrm{~km} / \mathrm{s}$ for a $b=3$ model. $b$, the bias parameter, is a measure of the ratio of the galaxy-galaxy correlation function to the mass-mass correlation function. In the texture model, we also find $M \propto v_{c i r c}^{\gamma}$ with $\gamma \simeq 3.85$ for objects of mass $10^{10}-10^{15} M_{\odot}$, consistent with the FaberJackson and Fisher-Tully relationship. The mass multiplicity function, predicted by the analytical theory, agrees with both the slope and the amplitude of the Schechter luminosity function (Gooding et al. 1991) for the same assumed mass-to-light ratio, $100 M_{\odot} / L_{\odot}$ for an $L_{*}$ galaxy.

Using sophisticated numerical codes that include gravitational physics, hydrodynamics and radiation physics developed by Renyue Cen, we have made detailed simulations of galaxy formation in the texture model. These same codes have been used to study galaxy formation in other cosmological scenarios. The initial conditions for these simulations were generated by following the evolution of a coupled scalar field in the expanding universe (Spergel et al. 1990), together with the appropriate Green's function for the growth of structure (Park et al. 1991).

The texture model had several significant success in these hydrodynamical simulation (Cen et al. 1991): (1) the "galaxy" correlation function was significantly enhanced relative to the dark matter correlation function; (2) the era of galaxy formation began significantly earlier than in the $(\mathrm{CDM}+\mathrm{HZ})$ model. This alleviates the embarrasment caused by high redshift quasars (with small Gunn-Peterson absorption) and the dense spheroids observed in normal galaxies. The early object formation in the texture scenario is consistent with analytical estimates (Gooding et al. 1991); (3) the simulations reproduce a quite plaussible galaxy mass function, consistent with the observed number of galaxies and the observed shape of the mass function, again confirming our analytical estimates. It is quite interesting to note that the galaxy mass distributions found in the hydrodynamical simulations are consistent with observations of $(M / L)_{b}=4$ and $(M / L)_{\text {tot }}=70$. These numbers are what is observed in galactic disks and globular clusters on the one hand and, for $M / L$, in binary pairs of galaxies on the other. Yet even though the total $M / L$ far short of the connonical value $(M / L)=1400 h$ thought to be required to produce a flat universe, the universe computed in the simulation is by construction flat. This singular success is a measure of the physical bias occasioned by a careful treatment of the cooling and heating processes.

Large-scale numerical simulations that include only gravitational physics also indicate some promising aspects of the texture model (Park et al. 1991): we reproduce the observed galaxy-galaxy correlation function, find clusters of galaxies to be significantly clustered on a scale of $20-50 h^{-1}$ $\mathrm{Mpc}$ and coherent structures of over $50 \mathrm{~h}^{-1} \mathrm{Mpc}$ in the galaxy distribution. The large scale streaming motions observed are in good agreement with the observations: the average magnitude of the velocity field smoothed over $30 \mathrm{~h}^{-1} \mathrm{Mpc}$ is $430 \mathrm{~km} \mathrm{~s}^{-1}$. Global texture produces a cosmic Mach number which is compatible with observation. We also see significant evolution of clusters at low redshift. The most striking potential problem for the scenario, too high velocity dispersions in clusters, is discussed in section 3 .

\section{Cluster Formation in the Texture Model}

Using the spherical collapse model, we have been able to compute the predicted velocity distribution function of the texture model. In principle, this should be an important prediction and potentially a definitive test of the model. In this section, we review the observational data (optical velocity dispersions, $\mathrm{X}$-ray measurements and observations of gravitational lensing). Unfortunately, uncertainties in the data do not permit us to make definitive statements about the successes and failures of the texture model.

Observational of gravitational lensing of background galaxies is a powerful probe of a cluster potential. Unlike optical and X-ray observations, lensing directly probes the distribution of matter in the cluster. Tyson et al. (1990) reports the velocity dispersion of two clusters with this method, and in both cases the calculated velocity dispersion is lower than that estimated by sampling the cluster galaxies. However, the high velocity dispersions, $1100-1500 \mathrm{~km} / \mathrm{s}$, pose a difficulty for standard CDM model. High dispersion clusters apparently exist at large redshifts as one of the clusters resides at a $z=0.46$. Tyson (1991) and his collaborators have continued their deep CCD imaging program and detected lensed arcs in numerous rich clusters and have found several clusters 


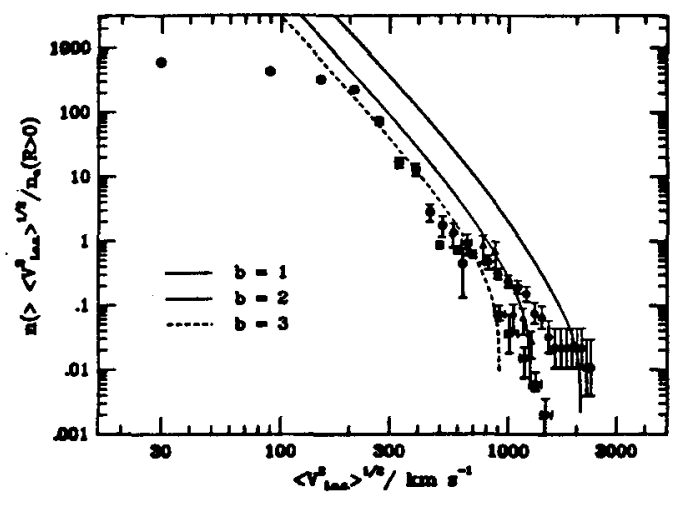

The solid lines in Figure 1 show the predictions of the texture model for the two initial velocity profiles and for different biases (normalizations). The the triangle show the cluster temperature function derived by Henry and Arnaud (1991). The open circles show the velocity dispersion function in the CfA redshift survey inferred by Moore (1991). The asterisks show the velocity distribution of the sample of Abell clusters used by Frenk et al (1990) [From Bartlett et al. 1991]

with velocity dispersions of upwards of $1800 \mathrm{~km} / \mathrm{s}$. The existence of these clusters is problematic for the standard CDM model and for texture models with $b>2$. A statistically complete sample of clusters whose potential have been measured through gravitational lensing will provide a definitive test of the texture model.

The texture model predicts the existence of high-velocity dispersion clusters at moderate redshift. Gunn and Dressler have reported that they have found several clusters that appear to have high velocity dispersion at moderate redshift,$z \sim 0.5$ ). Peebles et al. (1989) have argued that standard CDM cannot account for these objects. Kaiser (1991) has suggested that the existence of these clusters indicates little evolution in the velocity dispersion distribution from moderate redshifts to the present. This is difficult to understand in standard CDM if Press-Schechter is an accurate representation of the distribution function. In contrast clusters in the texture scenario formed earlier; we expect the distribution function to show less evolution than standard CDM. Figure 3 demonstrates that this is indeed the case. However, the current data does not permit us to make definitive statements about the viability of the texture model, but it does suggest the importance of observations of clusters as a probe of the origin of large scale structure.

\section{Rare Objects as a Probe of Primordial Fluctuations}

This non-Gaussian nature should be apparent in the predicted large scale structure. Recently, strong evidence for a non-Gaussian, positively skewed probability distribution for the galaxy field on large scales has been found by Efstathiou et al. (1990). In the distributions of galaxy counts in cells, there is a statistically significant excess of cells with high galaxy populations over the number predicted to occur with an underlying Gaussian galaxy distribution which has $\sigma^{2}$ chosen to match to the observations. Such a non-Gaussian distribution might be produced by nonlinear evolution from an initially Gaussian density field, as described in Peebles (1980). However in the inflation plus CDM theory, the variances on these scales are too small to produce significant nonlinearity, and the effect is minimal. We note that in the texture theory there is already significant positive skewness in the linear density field, so that even without nonlinear effects (which may after all be reduced by biasing) the theory may fit observation. The non-Gaussian excesses are caused, as pointed out in Efstathiou et al. (1990) by a few known structures in the galaxy distribution, discussed in detail by Saunders et al. (1991), and the number of the excess high-population cells is necessarily quite small due to the limited survey size, though statistically significant.

For comparison, we calculate the predicted number density of objects which resemble these known overdense structures, and in particular how many we would expect to see in the survey volume. 


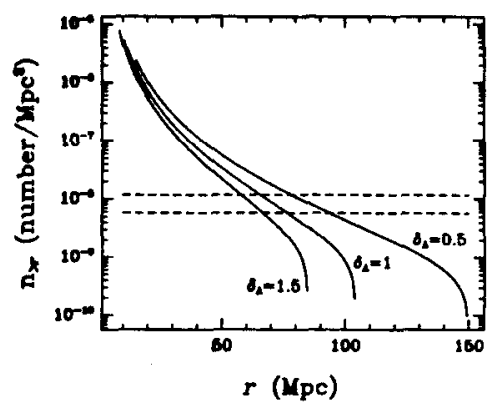

Figure 2 shows the number densities of objects of various $\delta$ with radius larger than a given $r$, as a function of $r$ [From Gooding et al. 1991b.]

Figure 2 shows that a few rare objects are expected to have formed on large scales in the texture model. For example, the number density of structures with $\delta \sim 0.5$ with radius larger than $\sim 80 \mathrm{Mpc}$ $(h=0.5)$ in our calculation suggests we should find, on average, $\sim 2$ such structures in the biggest survey box in Saunders et al. (1991). This is exactly what they find, suggesting the apparent nonGaussian features found in these surveys might have a simple explanation in the texture cosmogony - the giant superclusters are seeded by rare, large textures. We invite the reader to compare the predictions of Figure 2 with the density maps of Saunders et al. (1991).

\section{ACKNOWLEDGEMENTS}

This research is supported in part by NSF AST88-58145 (PYI) and an A.P. Sloan Fellowship. I would like to acknowledge my collaborators, Neil Turok, Jim Bartlett, Renyue Cen, Andy Gooding, Jeremiah Ostriker and Changbom Park.

\section{REFERENCES}

Bartlett, J., Gooding, A.K. and Spergel, D.N. 1991, submitted to Ap. J.

Cen, R. Y., Ostriker, J. P., Spergel, D. N., and Turok, N. 1991, to appear in Ap. J..

Frenk, C., White, S.D.M., Efstathiou, G. \& Davis, M. 1990, Ap.J., 351, 10.

Gooding, A. K., Spergel, D. N., Turok, N. 1991a, Ap. J. 372, L5.

Gooding, A.K., Park, C.B., Spergel, D.N., Turok, N. and Gott, J.R., III 1991b, submitted to Ap. $J$.

Gunn, J. E., and Gott, J. R. 1986, Ap. J. 176, 1.

Henry, J.P. and Arnaud, K.A. 1991 Ap. J., 372, 410.

Kaiser, N. 1991, CITA preprint

Moore, B. 1991, private communication

Park, C., Spergel, D. N., and Turok, N. 1991, Ap. J. 372, L53.

Peebles, P.J.E., Daly, R. and Juskiewicz, R. 1989, Ap. J., 347, 563.

Peebles, P.J.E. 1980, The Large Scale Structure of the Universe (Princeton: Princeton University Press)

Saunders, W., Frenk, C. S., Rowan-Robinson, M., Efstathiou, G., Lawrence, A., Kaiser, N., Ellis, R. S., Crawford, J., Xia, X., and Parry, I. 1991 Nature 349, 32.

Spergel, D. N., Turok, N., Press, W. H., and Ryden, B. S. 1991, Phys.Rev. D 431038.

Toth, G. and Ostriker, J.P. 1991, submitted to $A p$. J.

Turok, N. 1989, Phys. Rev. Lett., 63, 2625.

Turok, N., and Spergel, D. N. 1990, Phys. Rev. Lett. 64, 2736.

Tyson, J.A., Valdes, F. and Wenk, R.A. 1990, Ap. J., 349, L1.

Tyson, J.A. 1991, private communication 